\title{
DEREVERBERATION PERFORMANCE OF RIGID AND OPEN SPHERICAL MICROPHONE ARRAYS: THEORY \& SIMULATION
}

\author{
Daniel P. Jarrett ${ }^{\dagger}$,Emanuël A. P. Habets ${ }^{\star}$, Mark R. P. Thomas ${ }^{\dagger}$, Nikolay D. Gaubitch ${ }^{\dagger}$, Patrick A. Naylor ${ }^{\dagger}$ \\ $\dagger$ Dept. of Electrical \& Electronic Engineering, Imperial College London, UK \\ email: \{daniel.jarrett05, mark.r.thomas02,ndg, p.naylor\} @imperial.ac.uk \\ * International Audio Laboratories Erlangen, University of Erlangen-Nuremberg, Germany \\ email: emanuel.habets@audiolabs-erlangen.de
}

\begin{abstract}
Linear microphone arrays have been extensively used for dereverberation. In this paper we look at the dereverberation performance of two types of spherical microphone array: the open array (microphones suspended in free space) and the rigid array (microphones mounted on a rigid baffle). Dereverberation is performed in the spherical harmonic domain using a technique similar to the commonly used delay-and-sum beamformer (DSB). We analyse the theoretical performance with respect to the direct-to-reverberant ratio (DRR), and we also present simulation results obtained using a simulation tool for spherical arrays. The performance of the spherical DSB is found to increase with the radius of the sphere, and to be 1-2 $\mathrm{dB}$ higher for the rigid array. These results serve as a baseline for evaluating the performance of future dereverberation algorithms for spherical arrays.
\end{abstract}

Index Terms - Dereverberation, microphone array

\section{INTRODUCTION}

When a speech signal is captured in an enclosed environment such as an office room or an auditorium, its quality is affected by reverberation and ambient noise $[1,2]$. Reverberation can have detrimental effects on the perceived quality of speech and in some more severe cases it can cause loss of intelligibility. Reverberation also reduces the performance of applications such as speech recognition and speaker identification [3]. Consequently, dereverberation, the removal of the effects of room acoustics, has become an important area of signal processing research [2].

Several metrics have been developed to quantify the effects of reverberation and those of dereverberation algorithms. Most of these are the result of extensive research on the topics of intelligibility of music and speech in reverberant environments in the fields of psychoacoustics and architectural acoustics. Existing objective measures are mainly based on the room impulse response (RIR) [1,2]. An important class of RIR-based approaches measures the energy of the direct path signal relative to other features of the RIR [1]. Here we employ the direct-to-reverberant ratio (DRR) as a performance metric [2]; it is defined as the ratio of the energy of the direct signal component to the energy of the reflected signal components.

Beamforming is a fundamental multi-microphone technique, where all microphone signals are filtered and summed to achieve spatial selectivity. Here, we focus particularly on the use of a beamformer for sound acquisition in reverberant environments, where beamformers have shown particular promise [4].

Previous studies of dereverberation using microphone arrays have focused on linear arrays, e.g. [5]. However spherical arrays, where microphones are arranged in a spherical configuration either in free space (an open sphere) or on a rigid baffle (a rigid sphere), have recently become a topic of interest due to their ability to analyse sound fields in three dimensions, using an efficient spherical harmonic framework.

In this paper, we derive an expression for a spherical microphone array's expected dereverberation performance in terms of the DRR (for an analysis in terms of speech intelligibility see [6]). The expression for the scattering due to a rigid sphere is formulated in the spherical harmonic domain (SHD). We therefore also develop our DRR expression in this domain, thus allowing us to analyse both open and rigid arrays. We perform dereverberation using a so-called delayand-sum beamformer (DSB) in the SHD, which for the open sphere case is equivalent to the well-known time/frequency domain DSB. We use tools from statistical room acoustics (SRA) in order to find the expected DRR improvement at the beamformer output compared to a microphone placed at a position equivalent to the centre of the sphere.

This paper is organised as follows: in Section 2, we introduce some spherical harmonic concepts and give the expression for a DSB in the SHD; in Section 3, we derive a theoretical expression for the DRR improvement achieved with a spherical DSB; in Section 4, we describe the simulation method used to determine the room transfer functions which model our reverberant room; and in Section 5 we present both theoretical and simulation results for open and rigid arrays. 


\section{DELAY-AND-SUM BEAMFORMER IN THE SPHERICAL HARMONIC DOMAIN}

We consider the sound pressure field $p(k, \mathbf{r})$ at a position $\mathbf{r}=(r, \Omega)$ (in spherical coordinates) on the surface of a sphere of radius $r$, where $k$ is the wavenumber. Fig. 1 shows a diagram of the room layout. The spherical Fourier transform of this field is given by [7, p. 192]

$$
p_{n m}(k, r)=\int_{\Omega \in S^{2}} p(k, \mathbf{r}) Y_{n m}^{*}(\Omega) d \Omega,
$$

where $n$ is the order, $m$ is the degree, $\int_{\Omega \in S^{2}} d \Omega \triangleq \int_{0}^{2 \pi} \int_{0}^{\pi} \sin \theta d \theta d \phi$, the basis functions $Y_{n m}$ are the spherical harmonics, and $(\cdot)^{*}$ denotes the complex conjugate. The spherical harmonics $Y_{n m}(\Omega)$ of order $n$ and degree $m$, are given by [7, p. 190]

$$
Y_{n m}(\Omega)=\sqrt{\frac{(2 n+1)}{4 \pi} \frac{(n-m) !}{(n+m) !}} P_{n m}(\cos \theta) e^{i m \phi},
$$

where $P_{n m}$ is the associated Legendre function and $i=\sqrt{-1}$. The spherical harmonics satisfy the following orthogonality property [7, p. 191]:

$$
\int_{\Omega \in S^{2}} Y_{n m}(\Omega) Y_{p q}^{*}(\Omega) d \Omega=\delta_{n p} \delta_{m q},
$$

where $\delta$ is defined by

$$
\delta_{i j}=\left\{\begin{array}{lc}
1, & \text { if } i=j \\
0, & \text { otherwise }
\end{array}\right.
$$

A sum of spherical harmonics over all degrees $m$ can be simplified using the spherical harmonics addition theorem:

$$
\frac{2 n+1}{4 \pi} P_{n}\left(\cos \Theta_{a, b}\right)=\sum_{m=-n}^{n} Y_{n m}^{*}\left(\Omega_{a}\right) Y_{n m}\left(\Omega_{b}\right)
$$

where $\Theta_{a, b}$ is the angle between $\Omega_{a}$ and $\Omega_{b}$.

The inverse spherical Fourier transform of a sound field $p$ is given by the sum of all spherical Fourier coefficients $p_{n m}$ multiplied by the appropriate spherical harmonic $Y_{n m}$ :

$$
p(k, \mathbf{r})=\sum_{n=0}^{\infty} \sum_{m=-n}^{n} p_{n m}(k, r) Y_{n m}(\Omega) .
$$

The spherical Fourier transform of the sound field due to a unit amplitude plane wave impinging on a spherical microphone array with an arrival direction $\Omega_{s}$ is given by ${ }^{1}[9,10]$

$$
p_{n m}(k, r)=4 \pi(-i)^{n} b_{n}(k r) Y_{n m}^{*}\left(\Omega_{s}\right),
$$

where $b_{n}$ is the mode strength for an order $n$. For an open sphere, the mode strength is given by the spherical Bessel

\footnotetext{
${ }^{1}$ We adopt the sign convention commonly used in physics, as in $[7,8]$.
}

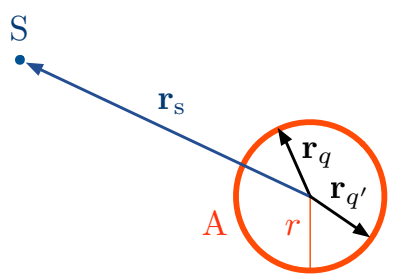

Fig. 1. Room layout in two dimensions (third dimension is not shown), showing a source $\mathrm{S}$ and a spherical microphone array A. Two sample microphones are located at $\mathbf{r}_{q}=\left(r, \Omega_{q}\right)$ and $\mathbf{r}_{q^{\prime}}=\left(r, \Omega_{q^{\prime}}\right)$, and a source is located at $\mathbf{r}_{\mathrm{s}}=\left(r_{\mathrm{s}}, \Omega_{\mathrm{s}}\right)$.

function $b_{n}(k r)=j_{n}(k r)$. For a rigid sphere, we add a term to account for the scattering [7, p. 228]:

$$
b_{n}(k r)=j_{n}(k r)-\frac{j_{n}^{\prime}(k r)}{h_{n}^{(1)^{\prime}}(k r)} h_{n}^{(1)}(k r),
$$

where $h^{(1)}$ is the spherical Hankel function of the first kind, and $h^{(1)^{\prime}}$ is its first derivative with respect to $k r$. In the spatial domain, this sound field is given by the inverse Fourier transform of (7):

$$
p(k, \mathbf{r})=\sum_{n=0}^{\infty} \sum_{m=-n}^{n} 4 \pi(-i)^{n} b_{n}(k r) Y_{n m}^{*}\left(\Omega_{s}\right) Y_{n m}(\Omega) .
$$

Computing the spherical Fourier transform of a sound field using the expression in (1) requires a continuous sensor, however in practice we use a number of discrete microphones at positions $\mathbf{r}_{q}=\left(r, \Omega_{q}\right), q=1, \ldots, Q$ (where $Q$ denotes the number of microphones) and approximate the integral using a quadrature sum:

$$
p_{n m}(k, r)=\sum_{q=1}^{Q} a_{q}(k) p\left(k, \mathbf{r}_{q}\right) Y_{n m}^{*}\left(\Omega_{q}\right) .
$$

With suitable quadrature weights $a_{q}$, this approximation is exact provided the microphones are sufficient in number and suitably positioned. Further details regarding the choice of microphone positions and weights can be found in $[11,12]$.

The output of an array can be expressed in the spherical harmonic domain as:

$$
y(k, r)=\sum_{n=0}^{\infty} \sum_{m=-n}^{n} p_{n m}(k, r) w_{n m}^{*}(k),
$$

where $w_{n m}$ are the beamforming weights. For a delay-andsum beamformer (DSB) steered in a direction $\Omega_{1}$, assuming plane-wave incidence, the weights are given by [13]

$$
w_{n m}^{*}(k)=i^{n} b_{n}^{*}(k r) Y_{n m}\left(\Omega_{1}\right) .
$$




\section{DIRECT-TO-REVERBERANT RATIO}

The expected DRR improvement at the output of a DSB compared to the DRR at a single microphone located at the same position as the centre of the spherical array is defined as:

$$
E\{\hat{\gamma}(r)\}=10 \log _{10}\left(\frac{E\left\{\gamma_{\mathrm{DSB}}(r)\right\}}{E\left\{\gamma^{\prime}\right\}}\right),
$$

where $E\{\cdot\}$ denotes the spatial expectation, $\gamma_{\mathrm{DSB}}$ is the DRR at the output of the DSB and $\gamma^{\prime}$ is the DRR at a single microphone located at the same position as the centre of the array (with no array present). We will first look to estimate $E\left\{\gamma^{\prime}\right\}$, followed by $E\left\{\gamma_{\mathrm{DSB}}(r)\right\}$.

As in SRA theory [1], we make the assumption that the room transfer function (RTF) $H\left(k, \mathbf{r}_{q}\right)$ from the location of the source $\mathbf{r}_{\mathrm{s}}$ to the $q^{\text {th }}$ microphone location $\mathbf{r}_{q}$ can be expressed in terms of a direct component $H_{\mathrm{d}}$ and a reverberant component $H_{\mathrm{r}}$ :

$$
H\left(k, \mathbf{r}_{q}\right)=H_{\mathrm{d}}\left(k, \mathbf{r}_{q}\right)+H_{\mathrm{r}}\left(k, \mathbf{r}_{q}\right),
$$

where the dependence on $\mathbf{r}_{\mathrm{s}}$ has been omitted for simplicity. We also assume that the direct and reverberant components are uncorrelated, and that the power spectral density of the RTF $H$ is therefore given by

$$
E\left\{\left|H\left(k, \mathbf{r}_{q}\right)\right|^{2}\right\}=\left|H_{\mathrm{d}}\left(k, \mathbf{r}_{q}\right)\right|^{2}+E\left\{\left|H_{\mathrm{r}}\left(k, \mathbf{r}_{q}\right)\right|^{2}\right\} .
$$

The direct component is given by [7, p. 227]

$$
\begin{aligned}
& H_{\mathrm{d}}\left(k, \mathbf{r}_{q}\right)= \\
& \quad \sum_{n=0}^{\infty} \sum_{m=-n}^{n} b_{n}(k r) k i h_{n}^{(1)}\left(k r_{\mathrm{s}}\right) Y_{n m}^{*}\left(\Omega_{s}\right) Y_{n m}\left(\Omega_{q}\right),
\end{aligned}
$$

where $r_{\mathrm{s}}$ is the distance between the centre of the array and the source. Using the spherical harmonic addition theorem from (5), this simplifies to

$$
\begin{aligned}
& H_{\mathrm{d}}\left(k, \mathbf{r}_{q}\right)= \\
& \quad \sum_{n=0}^{\infty} b_{n}(k r) k i h_{n}^{(1)}\left(k r_{\mathrm{s}}\right) \frac{(2 n+1)}{4 \pi} P_{n}\left(\cos \Theta_{\mathrm{s}, q}\right) .
\end{aligned}
$$

For an open sphere [i.e. $\left.b_{n}(k r)=j_{n}(k r)\right]$ this is equal to the familiar free-space Green's function [7, p. 259]:

$$
H_{\mathrm{d}}\left(k, \mathbf{r}_{q}\right)=\frac{e^{i k\left\|\mathbf{r}_{q}-\mathbf{r}_{\mathrm{s}}\right\|}}{4 \pi\left\|\mid \mathbf{r}_{q}-\mathbf{r}_{\mathrm{s}}\right\|} .
$$

The spatial cross-correlation between the pressure due to a diffuse sound field at two microphones with positions $\mathbf{r}_{q}$ and $\mathbf{r}_{q^{\prime}}$ is found to be proportional to $C\left(k, \mathbf{r}_{q}, \mathbf{r}_{q^{\prime}}\right)$ which is given by (see Appendix for derivation)

$$
\begin{aligned}
C\left(k, \mathbf{r}_{q}, \mathbf{r}_{q^{\prime}}\right) & =\Re\left\{E\left\{p\left(k, \mathbf{r}_{q}\right) p^{*}\left(k, \mathbf{r}_{q^{\prime}}\right)\right\}\right\} \\
& =\sum_{n=0}^{\infty}\left|b_{n}(k r)\right|^{2}(2 n+1) P_{n}\left(\cos \Theta_{q, q^{\prime}}\right) .
\end{aligned}
$$

In the open sphere case, for any frequency above the Schroeder frequency [1], the mean square reverberant component is given by $[5,14]$

$$
E\left\{\left|H_{\mathrm{r}}\left(k, \mathbf{r}_{q}\right)\right|^{2}\right\}=\frac{1-\alpha}{\pi A \alpha},
$$

where $\alpha$ is the average wall absorption coefficient (which in real rooms is frequency dependent however for simplicity will be assumed to be frequency independent here) and $A$ is the total wall surface area. The equations for the rigid sphere case, which must account for scattering, will be derived in a future paper. The cross-correlation between the reverberant components of the RTF is therefore given by

$$
\begin{aligned}
& E\left\{H_{\mathrm{r}}\left(k, \mathbf{r}_{q}\right) H_{\mathrm{r}}^{*}\left(k, \mathbf{r}_{q^{\prime}}\right)\right\}= \\
& \quad \frac{1-\alpha}{\pi A \alpha} \sum_{n=0}^{\infty}\left|b_{n}(k r)\right|^{2}(2 n+1) P_{n}\left(\cos \Theta_{q, q^{\prime}}\right) .
\end{aligned}
$$

Using (18) and (21), the expected DRR for a microphone placed at the centre of the array $\left(\mathbf{r}_{q}=\mathbf{0}\right)$ is given by

$$
\begin{aligned}
E\left\{\gamma^{\prime}\right\} & =\frac{\sum_{k \in \mathcal{K}}\left|H_{\mathrm{d}}(k, \mathbf{0})\right|^{2}}{\sum_{k \in \mathcal{K}} E\left\{\left|H_{\mathrm{r}}(k, \mathbf{0})\right|^{2}\right\}} \\
& =\frac{A \alpha}{(1-\alpha) 16 \pi \|\left.\mathbf{r}_{\mathrm{s}}\right|^{2}},
\end{aligned}
$$

where $\mathcal{K}$ denotes a set of discrete values of wavenumber $k$.

Now we develop an expression for $E\left\{\gamma_{\mathrm{DSB}}(r)\right\}$. If we consider an $N^{\text {th }}$ order spherical microphone array, based on (11) and (12), the transfer function $\bar{H}(k, r)$ from the source to the DSB output is given by

$$
\begin{array}{r}
\bar{H}(k, r)=\sum_{n=0}^{N} \sum_{m=-n}^{n}\left(\int_{\Omega \in S^{2}} H(k, \mathbf{r}) Y_{n m}^{*}(\Omega) d \Omega\right) \\
i^{n} b_{n}^{*}(k r) Y_{n m}\left(\Omega_{1}\right) .
\end{array}
$$

The mean square beamformer output can also be expressed in terms of a direct component $\bar{H}_{\mathrm{d}}$ and reverberant component $\bar{H}_{\mathrm{r}}$ :

$$
E\left\{|\bar{H}(k, r)|^{2}\right\}=\left|\bar{H}_{\mathrm{d}}(k, r)\right|^{2}+E\left\{\left|\bar{H}_{\mathrm{r}}(k, r)\right|^{2}\right\} .
$$

Using (24) and (17), the direct component is found to be (see Appendix for derivation):

$$
\begin{aligned}
& \left|\bar{H}_{\mathrm{d}}(k, r)\right|^{2}=\mid \sum_{n=0}^{N} \sum_{m=-n}^{n}\left(\int_{\Omega \in S^{2}} H_{\mathrm{d}}(k, \mathbf{r}) Y_{n m}^{*}(\Omega) d \Omega\right) \\
& \left.i^{n} b_{n}^{*}(k r) Y_{n m}\left(\Omega_{1}\right)\right|^{2} \\
& =\left.\left.\left|\sum_{n=0}^{N}\right| b_{n}(k r)\right|^{2} \frac{(2 n+1)}{4 \pi} k i^{n+1} h_{n}^{(1)}\left(k r_{\mathrm{s}}\right) P_{n}\left(\cos \Theta_{\mathrm{s}, 1}\right)\right|^{2} .
\end{aligned}
$$


If the look direction is the source direction, i.e. $\Omega_{\mathrm{s}}=\Omega_{1}$, then this reduces to:

$$
\left|\bar{H}_{\mathrm{d}}(k, r)\right|^{2}=\left.\left.\left|\sum_{n=0}^{N}\right| b_{n}(k r)\right|^{2} \frac{(2 n+1)}{4 \pi} k i^{n+1} h_{n}^{(1)}\left(k r_{\mathrm{s}}\right)\right|^{2}(27)
$$

The reverberant component is given by

$$
\begin{array}{r}
E\left\{\left|\bar{H}_{\mathrm{r}}(k, r)\right|^{2}\right\} \\
=E\left\{\mid \sum_{n=0}^{N} \sum_{m=-n}^{n}\left(\int_{\Omega \in S^{2}} H_{\mathrm{r}}(k, \mathbf{r}) Y_{n m}^{*}(\Omega) d \Omega\right)\right. \\
\left.\left.i^{n} b_{n}^{*}(k r) Y_{n m}\left(\Omega_{1}\right)\right|^{2}\right\} .
\end{array}
$$

The final expression is derived in the Appendix and given in (34).

Finally we obtain the DRR of the delay-and-sum beamformer:

$$
E\left\{\gamma_{\mathrm{DSB}}(r)\right\}=\frac{\sum_{k \in \mathcal{K}}\left|\bar{H}_{\mathrm{d}}(k, r)\right|^{2}}{\sum_{k \in \mathcal{K}} E\left\{\left|\bar{H}_{\mathrm{r}}(k, r)\right|^{2}\right\}},
$$

where $\left|\bar{H}_{\mathrm{d}}(k, r)\right|^{2}$ and $E\left\{\left|\bar{H}_{\mathrm{r}}(k, r)\right|^{2}\right\}$ are respectively given in (26) and (34). We can now evaluate the expected DRR improvement as defined in (13) using (23) and (29).

\section{SIMULATION METHOD}

We make use of a simulation method first proposed in [8]. This method allows us to simulate RTFs taking into account both the room reverberation and, in the case of the rigid sphere, the scattering introduced by the array.

The principle of the method is to model the RTFs as a sum of the responses from individual image sources, as in Allen \& Berkley's image method [15]. However, while in the image method the individual responses are the free-space Green's functions, here they are replaced with a spherical harmonic decomposition, as in (17), which allows us to account for scattering. The distance $r_{\mathrm{s}}$ in (17) is then the distance between the centre of the array and the image source, and the angle $\Theta_{\mathrm{s}, q}$ is the angle between the $q^{\text {th }}$ microphone and the image source. Further information on the method and its implementation, SMIRgen [16], is available in [8].

To illustrate the scattering effect, Fig. 2 shows two room transfer functions obtained using this method: one for a rigid sphere, and one for an open sphere, both of radius $2 \mathrm{~cm}$. The transfer functions have been limited to the direct path and a single first order reflection for clarity. It can be seen that the effects of scattering become more and more pronounced as frequency increases.

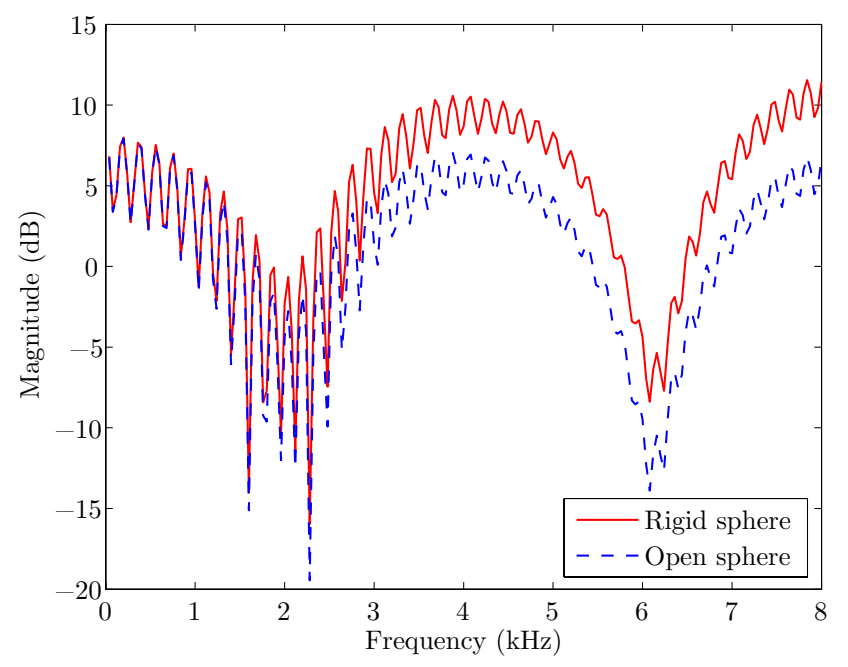

Fig. 2. A comparison of RTF magnitudes in the open and rigid sphere cases, for a microphone positioned on the near side (close to the source) of an array with radius $2 \mathrm{~cm}$.

\section{RESULTS}

In order to validate the theoretical expression derived for the DRR improvement which is achievable with a spherical DSB, and to gain some insight into the performance of a DSB for dereverberation, in this section we present simulation results alongside the theoretical results. Although the theoretical expression has not been derived for the rigid sphere, simulation results are shown to illustrate the advantage of the rigid sphere.

For our simulations we generated RTFs using the method described in Section 4. We modeled a room with dimensions $4 \times 5 \times 6.4 \mathrm{~m}$, as in $[5,14,17]$, in order to best approximate a diffuse sound field. A second order $(N=2)$ spherical array was used, with equiangular microphone positioning; we therefore used $4(N+1)^{2}=36$ microphones, however other more complex microphone configurations allow for a reduced number of microphones. The reverberation time $\mathrm{RT}_{60}$ was set to $500 \mathrm{~ms}$, thus giving an average wall absorption coefficient of $\alpha=0.2656$, and the sampling frequency was set to $8 \mathrm{kHz}$. In order to satisfy the conditions for a diffuse sound field [1], we considered frequencies well above the Schroeder frequency of $2000 \sqrt{\frac{0.5}{4 \cdot 5 \cdot 6.4}}=125 \mathrm{~Hz}$, specifically from 300 to $3400 \mathrm{~Hz}$, and both sources and microphones were kept at least half a wavelength from the walls of the room.

The spatial expectation was computed using an average over 50 source-array positions, using the approach in [14]: the array and source were kept in a fixed configuration (at a distance of $2 \mathrm{~m}$ from each other), which was then randomly rotated and translated. The reverberant component $H_{\mathrm{r}}$ of the RTFs was computed by subtracting the direct path from the reverberant RTFs. 


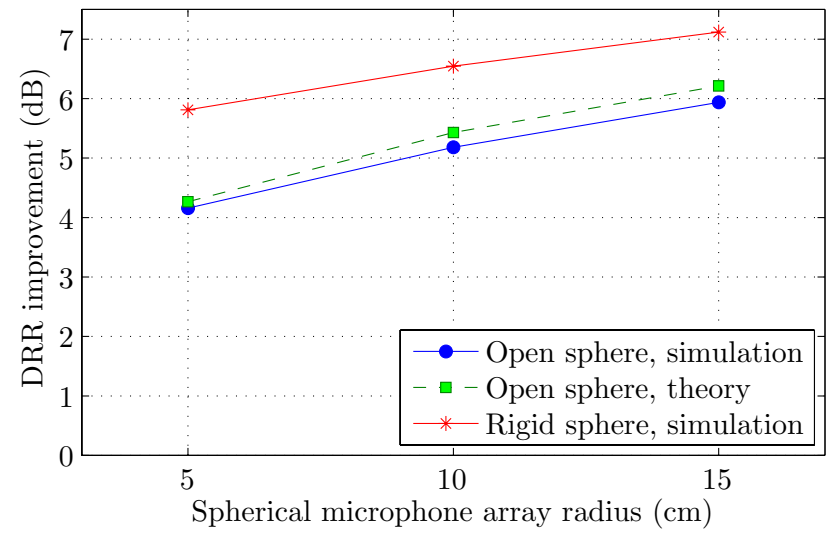

Fig. 3. DRR improvement as a function of the array radius, for a source-array distance of $2 \mathrm{~m}$.

The simulation results for both the open and rigid spheres, along with the theoretical results for the open sphere, are shown in Fig. 3, for arrays with a radius of 5, 10 and $15 \mathrm{~cm}$. A reasonably good match between the theoretical and simulation results is achieved.

It can be seen that the DRR improvement achieved increases with the array radius: as the sphere grows, the spatial diversity increases due to the larger separation between microphones. Our results are broadly similar to those obtained in [5]. Although more microphones are used here, the arrays are physically much smaller when compared to a linear array with a $20 \mathrm{~cm}$ separation between microphones, as in [5].

The results also show the improved dereverberation performance of a rigid array. This is consistent with the theoretical directivity index for a rigid array, which is higher than for an open array [13]. As we have a diffuse sound field, a higher directivity index yields a higher DRR.

\section{CONCLUSIONS}

In this paper we have derived a theoretical expression for the dereverberation performance of a spherical DSB with respect to DRR. We have compared the results from this expression to simulation results for open arrays, as well as presenting the simulation results for a rigid array which is found to have better performance. We find the performance is improved when choosing an array with a larger radius, as is the case for a linear array with larger spacing between microphones. The results obtained could serve as a baseline for evaluating the performance of more sophisticated dereverberation algorithms for spherical microphone arrays.

\section{REFERENCES}

[1] H. Kuttruff, Room Acoustics, 4th ed. Taylor \& Frances, 2000.
[2] P. A. Naylor and N. D. Gaubitch, Eds., Speech Dereverberation. Springer, 2010.

[3] A. Sehr, M. Zeller, and W. Kellermann, "Hands-free speech recognition using a reverberation model in the feature domain," in Proc. European Signal Processing Conf. (EUSIPCO), Florence, Italy, Sep. 2006.

[4] M. S. Brandstein and D. B. Ward, Eds., Microphone Arrays: Signal Processing Techniques and Applications. Berlin, Germany: Springer-Verlag, 2001.

[5] N. D. Gaubitch and P. A. Naylor, "Analysis of the dereverberation performance of microphone arrays," in Proc. Intl. Workshop Acoust. Echo Noise Control (IWAENC), 2005.

[6] Y. Peled and B. Rafaely, "Study of speech intelligibility in noisy enclosures using spherical microphones arrays," in Proc. Joint Workshop on HandsFree Speech Communication and Microphone Arrays (HSCMA), May 2008, pp. 160-163.

[7] E. G. Williams, Fourier Acoustics: Sound Radiation and Nearfield Acoustical Holography, 1st ed. Academic Press, 1999.

[8] D. P. Jarrett, E. A. P. Habets, M. R. P. Thomas, and P. A. Naylor, "Simulating room impulse responses for spherical microphone arrays," in Proc. IEEE Intl. Conf. on Acoustics, Speech and Signal Processing (ICASSP), May 2011.

[9] B. Rafaely, "Plane-wave decomposition of the pressure on a sphere by spherical convolution," J. Acoust. Soc. Am., vol. 116, no. 4, pp. 2149-2157, Oct. 2004.

[10] J. Meyer and G. Elko, "A highly scalable spherical microphone array based on an orthonormal decomposition of the soundfield," in Proc. IEEE Intl. Conf. on Acoustics, Speech and Signal Processing (ICASSP), vol. 2, May 2002, pp. 1781-1784.

[11] B. Rafaely, B. Weiss, and E. Bachmat, "Spatial aliasing in spherical microphone arrays," IEEE Trans. Signal Process., vol. 55, no. 3, pp. 1003-1010, Mar. 2007.

[12] J. R. Driscoll and D. M. Healy, "Computing Fourier transforms and convolutions on the 2-Sphere," Advances in Applied Mathematics, vol. 15, no. 2, pp. 202-250, 1994.

[13] B. Rafaely, "Phase-mode versus delay-and-sum spherical microphone array processing," IEEE Signal Process. Lett., vol. 12, no. 10, pp. 713-716, Oct. 2005.

[14] B. D. Radlović, R. Williamson, and R. Kennedy, "Equalization in an acoustic reverberant environment: robustness results," IEEE Trans. Speech Audio Process., vol. 8, no. 3, pp. 311-319, 2000.

[15] J. B. Allen and D. A. Berkley, "Image method for efficiently simulating small-room acoustics," J. Acoust. Soc. Am., vol. 65, no. 4, pp. 943-950, Apr. 1979.

[16] D. P. Jarrett. Spherical microphone array impulse response (SMIR) generator. [Online]. Available: http: //www.ee.ic.ac.uk/sap/smirgen/

[17] F. Talantzis and D. B. Ward, "Robustness of multichannel equalization in an acoustic reverberant environment," J. Acoust. Soc. Am., vol. 114, no. 2, pp. 833-841, 2003. 


\section{APPENDIX}

If we have a diffuse sound field, the spatial cross-correlation between the sound pressure at two positions $\mathbf{r}_{q}$ and $\mathbf{r}_{q^{\prime}}$ is proportional to:

$$
\begin{aligned}
C\left(k, \mathbf{r}_{q}, \mathbf{r}_{q^{\prime}}\right) & =\Re\left\{E\left\{p\left(k, \mathbf{r}_{q}\right) p^{*}\left(k, \mathbf{r}_{q^{\prime}}\right)\right\}\right\} \\
& =\frac{1}{4 \pi} \Re\left\{\int_{\Omega \in S^{2}} p\left(k, \mathbf{r}_{q}\right) p^{*}\left(k, \mathbf{r}_{q^{\prime}}\right) d \Omega\right\} \\
& =\frac{1}{4 \pi} \Re\left\{\int_{\Omega \in S^{2}} \sum_{n=0}^{\infty} \sum_{m=-n}^{n} 4 \pi(-i)^{n} b_{n}(k r) Y_{n m}^{*}(\Omega) Y_{n m}\left(\Omega_{q}\right) \sum_{n^{\prime}=0}^{\infty} \sum_{m^{\prime}=-n^{\prime}}^{n^{\prime}} 4 \pi i^{n^{\prime}} b_{n^{\prime}}^{*}(k r) Y_{n^{\prime} m^{\prime}}(\Omega) Y_{n^{\prime} m^{\prime}}^{*}\left(\Omega_{q^{\prime}}\right) d \Omega\right\}
\end{aligned}
$$

Using the orthogonality property of the spherical harmonics from (3), we obtain:

$$
\begin{aligned}
C\left(k, \mathbf{r}_{q}, \mathbf{r}_{q^{\prime}}\right) & =\frac{1}{4 \pi} \Re\left\{\sum_{n=0}^{\infty} \sum_{m=-n}^{n}(4 \pi)^{2}\left|b_{n}(k r)\right|^{2} Y_{n m}\left(\Omega_{q}\right) Y_{n m}^{*}\left(\Omega_{q^{\prime}}\right)\right\} \\
& =\frac{1}{4 \pi} \Re\left\{\sum_{n=0}^{\infty}(4 \pi)^{2}\left|b_{n}(k r)\right|^{2} \frac{2 n+1}{4 \pi} P_{n}\left(\cos \Theta_{q, q^{\prime}}\right)\right\} \\
& =\sum_{n=0}^{\infty}\left|b_{n}(k r)\right|^{2}(2 n+1) P_{n}\left(\cos \Theta_{q, q^{\prime}}\right)
\end{aligned}
$$

The direct component $\bar{H}_{\mathrm{d}}$ of the beamformer output $\bar{H}$ is given by:

$$
\begin{aligned}
\left|\bar{H}_{\mathrm{d}}(k, r)\right|^{2} & =\left|\sum_{n=0}^{N} \sum_{m=-n}^{n}\left(\int_{\Omega \in S^{2}} H_{\mathrm{d}}(k, \mathbf{r}) Y_{n m}^{*}(\Omega) d \Omega\right) i^{n} b_{n}^{*}(k r) Y_{n m}\left(\Omega_{\mathrm{l}}\right)\right|^{2} \\
& =\left|\sum_{n=0}^{N} \sum_{m=-n}^{n}\left(\int_{\Omega \in S^{2}}\left(\sum_{n=0}^{\infty} \sum_{m=-n}^{n} b_{n}(k r) k i h_{n}^{(1)}\left(k r_{\mathrm{s}}\right) Y_{n m}^{*}\left(\Omega_{s}\right) Y_{n m}(\Omega)\right) Y_{n m}^{*}(\Omega) d \Omega\right) i^{n} b_{n}^{*}(k r) Y_{n m}\left(\Omega_{\mathrm{l}}\right)\right|^{2} \\
& =\left|\sum_{n=0}^{N} \sum_{m=-n}^{n} b_{n}(k r) k i^{n+1} h_{n}^{(1)}\left(k r_{\mathrm{s}}\right) Y_{n m}^{*}\left(\Omega_{s}\right) b_{n}^{*}(k r) Y_{n m}\left(\Omega_{\mathrm{l}}\right)\right|^{2} \\
& =\left.\left.\left|\sum_{n=0}^{N}\right| b_{n}(k r)\right|^{2} \frac{(2 n+1)}{4 \pi} k i^{n+1} h_{n}^{(1)}\left(k r_{\mathrm{s}}\right) P_{n}\left(\cos \Theta_{\mathrm{s}, 1}\right)\right|^{2}
\end{aligned}
$$

The reverberant component $\bar{H}_{\mathrm{r}}$ is given by:

$$
E\left\{\left|\bar{H}_{\mathrm{r}}(k, r)\right|^{2}\right\}=E\left\{\left|\sum_{n=0}^{N} \sum_{m=-n}^{n}\left(\int_{\Omega \in S^{2}} H_{\mathrm{r}}(k, \mathbf{r}) Y_{n m}^{*}(\Omega) d \Omega\right) i^{n} b_{n}^{*}(k r) Y_{n m}\left(\Omega_{1}\right)\right|^{2}\right\}
$$

We can replace the integral in this equation with a quadrature sum as in (10):

$$
\begin{aligned}
& E\left\{\left|\bar{H}_{\mathrm{r}}(k, r)\right|^{2}\right\} \\
= & E\left\{\left|\sum_{n=0}^{N} \sum_{m=-n}^{n} \sum_{q=1}^{Q} a_{q} Y_{n m}^{*}(\Omega) H_{\mathrm{r}}\left(k, \mathbf{r}_{q}\right) i^{n} b_{n}^{*}(k r) Y_{n m}\left(\Omega_{1}\right)\right|^{2}\right\} \\
= & E\left\{\left|\sum_{n=0}^{N} i^{n} b_{n}^{*}(k r) \frac{2 n+1}{4 \pi} \sum_{q=1}^{Q} a_{q} P_{n}\left(\cos \Omega_{q, l}\right) H_{\mathrm{r}}\left(k, \mathbf{r}_{q}\right)\right|^{2}\right\} \\
= & E\left\{\sum_{n=0}^{N} \sum_{n^{\prime}=0}^{N} i^{n} b_{n}^{*}(k r)(-i)^{n^{\prime}} b_{n^{\prime}}(k r) \frac{2 n+1}{4 \pi} \frac{2 n^{\prime}+1}{4 \pi} \sum_{q=1}^{Q} \sum_{q^{\prime}=1}^{Q} a_{q} a_{q^{\prime}}^{*} P_{n}\left(\cos \Omega_{q, l}\right) P_{n^{\prime}}\left(\cos \Omega_{q^{\prime}, l}\right) H_{\mathrm{r}}\left(k, \mathbf{r}_{q}\right) H_{\mathrm{r}}^{*}\left(k, \mathbf{r}_{q^{\prime}}\right)\right\} \\
= & \sum_{n=0}^{N} \sum_{n^{\prime}=0}^{N} i^{n} b_{n}^{*}(k r)(-i)^{n^{\prime}} b_{n^{\prime}}(k r) \frac{2 n+1}{4 \pi} \frac{2 n^{\prime}+1}{4 \pi} \sum_{q=1}^{Q} \sum_{q^{\prime}=1}^{Q} a_{q} a_{q^{\prime}}^{*} P_{n}\left(\cos \Omega_{q, l}\right) P_{n^{\prime}}\left(\cos \Omega_{q^{\prime}, l}\right) E\left\{H_{\mathrm{r}}\left(k, \mathbf{r}_{q}\right) H_{\mathrm{r}}^{*}\left(k, \mathbf{r}_{q^{\prime}}\right)\right\}
\end{aligned}
$$

where the expression for $E\left\{H_{\mathrm{r}}\left(k, \mathbf{r}_{q}\right) H_{\mathrm{r}}^{*}\left(k, \mathbf{r}_{q^{\prime}}\right)\right\}$ is given in (22). 